\title{
Expression of discoidin domain receptor 1 tyrosine kinase on the human bronchial epithelium
}

\author{
O. Sakamoto*, M. Suga*, T. Suda\#, M. Ando*
}

Expression of discoidin domain receptor 1 tyrosine kinase on the human bronchial epithelium. O. Sakamoto, M. Suga, T. Suda, M. Ando. (C)ERS Journals Ltd 2001.

ABSTRACT: Discoidin domain receptor 1 (DDR1) tyrosine kinases constitute a novel family of receptors characterized by a unique structure in the ectodomain (discoidin-I domain). The DDR1 ligand is the extracellular matrix protein collagen.

To identify receptor tyrosine kinases (RTKs) involved in control of growth and differentiation of human bronchial epithelial (HBE) cells, a polymerase chain reactionbased search for RTKs in HBE cells was performed. DDR1 was the most abundant clone identified. Northern analysis detected a 3.6 kb DDR1 messenger ribonucleic acid (mRNA) expressed in HBE cells and transformed HBE lines, BET-1A and BEAS-2B. In addition, fluorescence-activated cell sorter (FACS) analyses using an anti-DDR1 antibody showed that DDR1 was expressed on HBE cells and two HBE lines.

Immunohistochemical staining using human bronchial tissue demonstrated that DDR1 was mainly expressed at the basolateral cell surface of the bronchial epithelium. Furthermore, immunostaining of type IV collagen, a major component of the basement membrane, clearly showed that the basement membrane was closely attached to the basal surface of the bronchial epithelium.

Since collagen binds to and activates discoidin domain receptor 1 tyrosine kinase, colocalization of discoidin domain receptor 1 and its ligand type IV collagen demonstrates a potential interaction of discoidin domain receptor 1 on the bronchial epithelium with type IV collagen. Further study of this interaction may define the functional significance of the collagen-discoidin domain receptor 1 signalling pathway in health and in disease.

Eur Respir J 2001; 17: 969-974.
*First Dept of Internal Medicine and ${ }^{\#}$ Dept of Cell Differentiation, IMEG, Kumamoto University School of Medicine, Kumamoto, Japan.

Correspondence: O. Sakamoto, First Dept of Internal Medicine, Kumamoto University School of Medicine, 1-1-1 Honjo, Kumamoto 860-0811, Japan.

Fax: 81963710582

Keywords: Bronchial epithelium discoidin domain receptor 1 receptor tyrosine kinase type IV collagen

Received: January 122000 Accepted after revision July 142000

This work was supported in part by Grants-in-Aid from the Ministry of Education, Science and Culture of Japan.
Receptor tyrosine kinases (RTKs) play a critical role in pleiotropic cell functions as components of signaltransduction pathways [1-3]. For example, many growth factor receptors are RTKs. After ligand binding, RTKs dimerize, leading to activation of intrinsic tyrosine kinase (TK) activity and autophosphorylation [2, 4]. In the airway epithelium, some RTKs have been shown to be involved in growth and differentiation. For example, both the epidermal growth factor receptor (EGFR) and hepatocyte growth factor receptor (HGFR) mediate growth signals $[5,6]$, and the RON TK promotes ciliary motility in response to its ligand, macrophage-stimulating protein [7]. However, how RTKs control cellular events in the airway epithelium is not understood.

To identify RTKs involved in various functions of airway epithelium, ribonucleic acid (RNA) from human bronchial epithelial (HBE) cells was prepared for polymerase chain reaction (PCR)-based cloning of protein tyrosine kinase (PTK)-related complementary deoxyribonucleic acid (cDNA) sequences. Sequencing of the cDNAs isolated showed that the discoidin domain receptor 1 (DDR1) TK was abundantly expressed on HBE cells. DDR1, which was recently identified by several investigators [8-14], possesses a motif in its extracellular domain, homologous to the slime mold Dictyostelium discoideum protein discoidin-I. Discoidin-I is a carbohydrate-binding lectin essential for $D$. discoideum cell aggregation [15]. In addition, discoidin-I domains are homologous to the constant regions of blood coagulation cofactors V and VIII [16] and to neuropilins and neurexins, which are involved in the development of the nervous system [17, 18]. Recently, the extracellular matrix protein collagen was shown to be a ligand that binds to and activates DDR1 [19-22]. However, the biological significance of this signalling pathway is not known.

To explore the functions of collagen-DDR 1 signalling pathway in the airway epithelium, it was determined which cell types express DDR1. It was also questioned whether collagen is expressed in close proximity to cells expressing DDR 1 . This study has shown that DDR1 is predominantly expressed on the basolateral cell surface of human bronchial epithelium. In addition, it is shown that type IV collagen of the basement membrane is closely attached to the basal surface of human bronchial epithelium. This study identifies a potential functional interaction of DDR 1 on the bronchial epithelium with its ligand type IV collagen within basement membranes. 


\section{Materials and methods}

\section{Reagents}

A synthetic polypeptide corresponding to the C-terminal 20 amino acids of human DDR 1 (amino acid residues $894-913$ ) and a rabbit polyclonal antibody against this synthetic polypeptide were obtained from Santa Cruz Biotechnology, Inc. (Santa Cruz, CA, USA). A mouse monoclonal anticollagen type IV antibody (clone CIV22) was obtained from DAKO (Glostrup, Denmark). Donkey antirabbit immunoglobulin-G (IgG) conjugated with horseradish peroxidase (HRP) and sheep antimouse IgG conjugated with HRP, were obtained from Amersham International (Amersham, UK). Goat antirabbit IgG conjugated with fluorescence isothiocyanate (FITC) was obtained from GIBCO BRL (Grand Island, NY, USA).

\section{Cells and cell cultures}

HBE cells were purchased from Clonetics (San Diego, CA, USA). Transformed HBE cell lines, BET-1A and BEAS-2B [23] were obtained from American Type Culture Collection (ATCC; Rockville, $\mathrm{MD}$, USA). These cells were cultured at $37^{\circ} \mathrm{C}$ in serumfree bronchial epithelial cell growth medium (BE-GM; SankoJunyaku, Tokyo, Japan) supplemented with growth factors according to the manufacturer's instructions. HBE cell lines were derived from transformed HBE cells and have been shown to be cultured in serum-free medium [23]. Since transforming growth factor- $\beta$ (TGF- $\beta$ ) in serum induces squamous differentiation of HBE cells [24], both primary HBE cells and HBE cell lines were cultured in serum-free BEGM. The surface of the culture dishes was coated with human placental collagen (Sigma, St. Louis, MO, USA) as described [25]. Third or fourth passage HBE cells were used.

\section{Preparation of degenerate oligonucleotide primers}

Two sets of degenerate oligonucleotide primers corresponding to the consensus sequences of kinase domains (sense primer, subdomain VI; antisense primer, subdomain IX) were synthesized. One set was directed to the sequence IHRDL (PTKI) and DVWSFG (PTKII), as described by WILKS [26], and the other was directed to the sequence HRDLA (TKI), HRDLAAR (TKII), and DVWSF/YG (TKIII). TKI-III primers were more degenerate than PTKI and II. The sequences of the primers are as follows: PTKI, $5^{\prime}$ - CGGATCCAC(A/C)GNGA(C/T)(C/T)T-3'; PTKII, 3'-CT(A/G)CA(C/G)ACCAGGA(A/T) ACCTTAAGG-5'; TKI, 5'-TAGTCGACA(C/T)(A/C) G(A/G)GA(C/T)(C/T)T(A/C/G)GC-3'; TKII, 5' TTGTCGACAC(A/C)G(A/G)GA(C/T)(C/T)T(C/G) GCNGCN(A/C)G-3'; TKIII, 3'-CT(A/G)CA(C/G) $\mathrm{ACC}(\mathrm{A} / \mathrm{T})(\mathrm{C} / \mathrm{G})(\mathrm{A} / \mathrm{G}) \mathrm{A}(\mathrm{A} / \mathrm{T}) \mathrm{ACCTTAAGGT}-5^{\prime}$.
Reverse transcriptase-polymerase chain reaction amplification of protein tyrosine kinase-related sequences

Poly(A) ${ }^{+}$RNA was obtained from HBE cells using a Micro-Fast Track Kit (Invitrogen Corporation, Carlsbad, CA, USA). The first-strand cDNA was synthesized from $1 \mathrm{mg}$ poly $(\mathrm{A})^{+}$RNA using the SUPERSCRIPT Preamplification System (GIBCO BRL). Two microlitres of the cDNA were used as the template for amplification. The cycling parameters were $1 \mathrm{~min}$ at $94^{\circ} \mathrm{C}, 2 \mathrm{~min}$ at $37^{\circ} \mathrm{C}$ and $3 \mathrm{~min}$ at $72^{\circ} \mathrm{C}$. In the first experiment, cDNA was primed with random hexamers and the amplification proceeded for 40 cycles with $1 \mathrm{mg}$ each of TKI and TKIII primers. The second experiment included a two-step amplification. The first amplification was performed for 20 cycles with $1 \mathrm{mg}$ each of PTKI and PTKII primers or TKI and TKIII primers. After the PCR products were purified using a CHROMA SPIN column (Clontech, Palo Alto, CA, USA), an additional 20 cycles of amplification were performed with $1 \mathrm{mg}$ each of TKII and TKIII primers. Amplified cDNA of $\sim 210 \mathrm{bp}$ was subcloned into the Bluescript vector (Stratagene, La Jolla, CA). Inserts of 70 randomly selected clones were sequenced by dideoxynucleotide chain termination, using an AutoRead sequence kit and the Automated Laser Fluorescent ALF DNA Sequencer (Pharmacia, Uppsala, Sweden).

\section{Northern blot analysis}

$\operatorname{Poly}(\mathrm{A})^{+}$RNA was isolated from HBE, BET-1A, and BEAS-2B cells by a Micro-Fast Track Kit (Invitrogen Corporation). Two micrograms of poly(A) ${ }^{+}$RNA was run on a formaldehyde gel, and Northern blots were prepared as described [27] using a Zeta-Probe membrane (Bio-Rad Lab., Richmond, CA, USA). Hybridization and washes were done according to the manufacturer's instructions. A human DDR1 cDNA probe corresponding to nucleotides $682-1,712$ of the published sequence [8] was [ $\alpha-{ }^{32}$ Phosphorus]labelled and hybridized to the blot.

Fluorescence-activated cell sorter analysis of human bronchial epithelial cells and human bronchial epithelial cell lines

HBE cells and HBE cell lines (BET-1A and BEAS2B) were detached from culture dishes with $5 \mathrm{mM}$ ethylenediamine tetraacetic acid (EDTA)/phosphatebuffered saline (PBS) (pH 7.4) and analysed by fluorescence-activated cell sorter (FACS). Briefly, the detached cells were washed with PBS and incubated with $70 \%$ ethanol on ice for $1 \mathrm{~h}$ to permeabilize and fix. After washing with staining medium containing 5\% foetal bovine serum (FBS) and $0.01 \%$ sodium azide in PBS, the cells were stained with anti-DDR 1 $\left(2 \mu \mathrm{g} \cdot \mathrm{mL}^{-1}\right)$, followed by FITC-conjugated antirabbit IgG. The cells were washed and suspended in staining medium containing propidium iodide. Stained cells were analysed by FACScan (Becton Dickinson Immunocytometry Systems, San Jose, CA, USA). Specific staining for DDR1 was confirmed by antigenpeptide blocking. 


\section{Immunohistochemistry}

Human bronchial tissues were obtained from five patients (two males, three females; two smokers, three nonsmokers) undergoing surgery for lung cancer. Ten subsegmental bronchi (diameter 3-6 $\mathrm{mm}$ ), which were macroscopically normal, were used in this study. It was confirmed that these bronchi were also microscopically normal by haematoxylin-eosin staining. These studies were approved by the Institutional Review Board of Kumamoto University. Immediately after the tissues were obtained, they were mounted in OCT 4583 embedding compound (Miles Inc., Elkhart, IN, USA) and frozen in liquid nitrogen. Serial cryostat sections (6 $\mu \mathrm{m}$ thick) cut in a MICROM cryomicrotome were air dried and fixed in acetone for $10 \mathrm{~min}$ at $4{ }^{\circ} \mathrm{C}$. After washing with PBS three times, cryostat sections were treated with $5 \mathrm{mM}$ orthoperiodic acid solution for $10 \mathrm{~min}$ as described [28] to block endogenous peroxidase activity. Sections stained with a rabbit polyclonal antibody against DDR $1\left(1 \mu \mathrm{g} \cdot \mathrm{mL}^{-1}\right)$ were analysed by the indirect immunoperoxidase method using HRPconjugated antirabbit IgG. Those stained with a mouse monoclonal anticollagen type IV antibody (clone CIV22, 1:100 dilution) were analysed by the same method using HRP-conjugated antimouse IgG. Peroxidase activity was visualized using 3,3'-diaminobenzidine (Dojindo, Kumamoto, Japan) as a substrate. To confirm the specific reactivity of anti-DDR1 with the DDR1 protein, one of the serial sections was incubated with a mixture of anti-DDR1 antibody and an excess molar ratio of antigen peptide and processed as described previously.

\section{Results}

Polymerase chain reaction amplification of tyrosine kinase-related complementary deoxyribonucleic acid

To identify RTKs involved in airway epithelial cell function, the strategy of PCR amplification described by WILKS [26] was used. In addition to the primers cited therein (PTKI and PTKII), more degenerate oligonucleotide primers (TKI-III) corresponding to the amino acid sequences HRDLAAR (subdomain VI) and DVWS $(\mathrm{F} / \mathrm{Y}) \mathrm{G}$ (subdomain IX) were prepared. The sense primers TKI and TKII were designed to preferentially clone RTK genes (HRDLAAR).

Random sequence analyses of PCR-amplified cDNA clones identified 13 distinct kinase-related genes, including nine RTKs and four nonreceptor PTKs (table 1). The profile of identified cDNAs differed according to the primers; more degenerate primers [TKII-III: sense primer, HRDLAAR; antisense primer, DVWS(F/Y)G] amplified a more extended family of kinase-related genes. Among RTKs identified in HBE cells, the incidence of DDR1 tyrosine kinase was highest (table 1).

Expression of discoidin domain receptor 1 in human bronchial epithelial cells and human bronchial epithelial cell lines

Based on the PCR results, expression of DDR1 tyrosine kinase in HBE cells and HBE cell lines
Table 1.-Profile of protein tyrosine kinases (PTKs) isolated by polymerase chain reaction-based cloning

\begin{tabular}{lcc}
\hline \multirow{2}{*}{ PTK } & \multicolumn{2}{c}{ Sense Primer } \\
\cline { 2 - 3 } & HRDLA & HRDLAAR \\
\hline DDR1* & 8 & 14 \\
EGFR* & 3 & 1 \\
IGF1R* & 1 & 4 \\
HGFR* & 1 & 2 \\
FGFR3* & & 5 \\
Tyro-3* & & 6 \\
ECK* & 1 & 4 \\
RYK* & 2 & 1 \\
EPH* & 4 & 3 \\
JAK1 & 1 & 5 \\
BRK & & 3 \\
c-ABL & & 1 \\
CSK & 21 & 49 \\
Total & & \\
\hline
\end{tabular}

*: Receptor PTKs. DDR1: discoidin domain receptor 1; EGFR: epidermal growth factor receptor; IGF1R: insulinlike growth factor receptor 1; HGFR: hepatocyte growth factor receptor; FGFR3: fibroblast growth factor receptor 3; ECK: epithelial cell kinase; RYK: related to tyrosine kinase; JAK1: janus kinase 1; BRK: breast tumour kinase; CSK: C-terminal Src kinase.

(BET-1A and BEAS-2B) was examined by Northern blot analysis. As shown in figure 1, a 3.6-kb DDR1 transcript was detected in HBE cells and HBE cell lines. To examine expression of DDR1 protein in these cells, FACS analyses were performed using an anti-DDR1 antibody. As shown in figure 2, FACS analyses show that HBE cells and HBE cell lines (BET-1A and BEAS2B) are uniformly positive for DDR1. The specificity of DDR1 staining was confirmed by the observation that the histogram of positive staining completely shifted to a basal level following peptide blocking.

Predominant expression of discoidin domain receptor 1 on the basolateral cell membrane of the bronchial epithelium

To identify specific cell types expressing DDR 1 in the bronchial epithelium, immunohistochemical staining of normal human bronchial tissues was performed using an anti-DDR1 antibody. In the bronchial epithelium, DDR1 was mainly expressed at the basolateral cell surface, suggesting predominant expression of DDR1 on the basolateral cell membrane of the bronchial epithelium (figure 3). Positive staining was completely blocked by an excess molar ratio of antigen peptide (figure 3), confirming the specific reactivity of antiDDR1 antibody with DDR1. There was no difference in DDR 1 expression between smokers and nonsmokers (data not shown).

Close attachment of discoidin domain receptor 1 and type IV collagen

Because DDR1 was predominantly expressed on the basolateral surface of the bronchial epithelium, the authors focused interaction of DDR 1 on the bronchial 


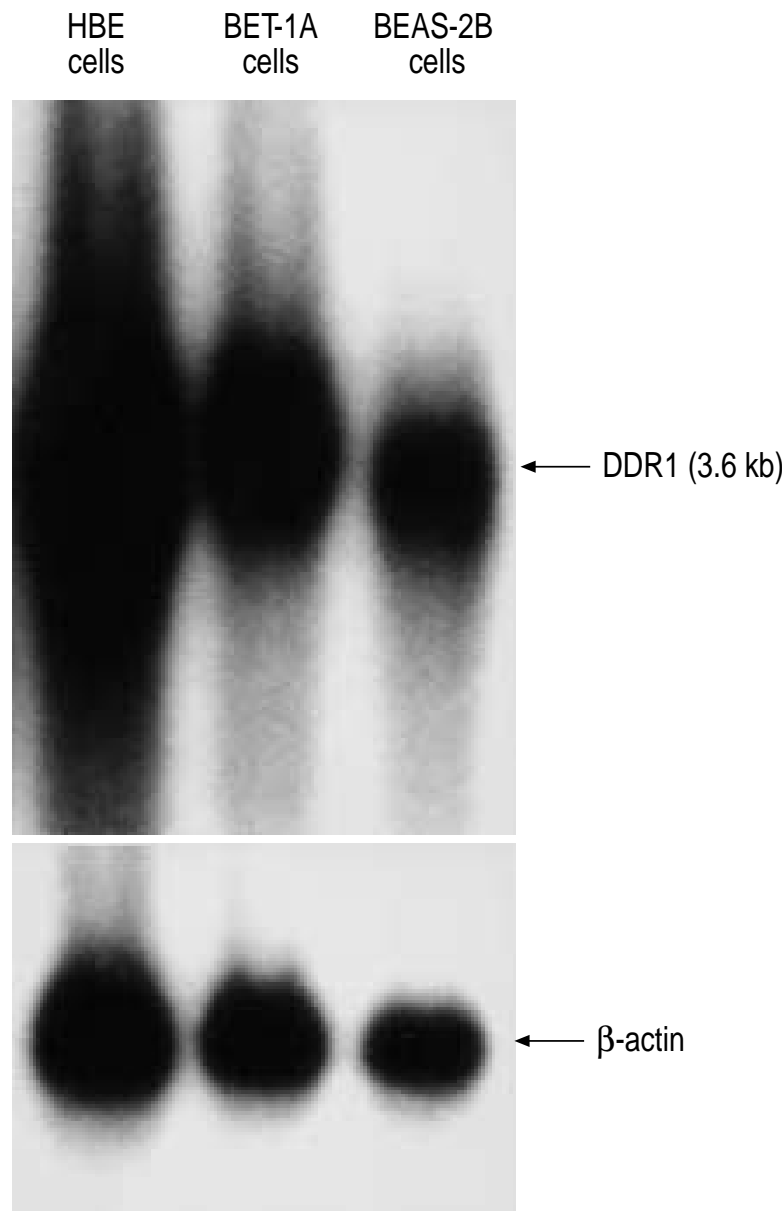

Fig. 1. - Northern blot of discoidin domain receptor 1 (DDR1) messenger ribonucleic acid (mRNA) in human bronchial epithelial (HBE) cells and HBE cell lines. The blot was hybridized with an $\left[\alpha-{ }^{32}\right.$ Phosphorous]-labelled DDR1 complementary deoxyribonucleic acid probe and exposed to film for 3 days. Expression of DDR1 mRNA was detected as a 3.6-kb band.

epithelium and basement membrane. As shown in figure 3, immunostaining of type IV collagen, which is a major component of the basement membrane, showed that type IV collagen of the basement membrane is closely attached to the basal surface of the bronchial epithelium.

\section{Discussion}

To identify RTKs involved in control of cellular responses of the airway epithelium, PCR-based cloning from $\mathrm{HBE}$ cells was performed. As shown in table 1, nine RTKs were identified in the study. Of these, the EGFR, insulin-like growth factor-1 receptor, and HGFR have been shown to be expressed in human bronchial epithelium where they transmit growth signals upon ligand stimulation [5, 6, 29]. In some epithelial cells, expression of fibroblast growth factor receptor-3, epithelial cell kinase, related to tyrosine kinase (RYK), and EPH has been reported [30-33]. These RTKs were identified in HBE cells in the screen; however, the function of these kinases in the bronchial epithelium has not been determined.
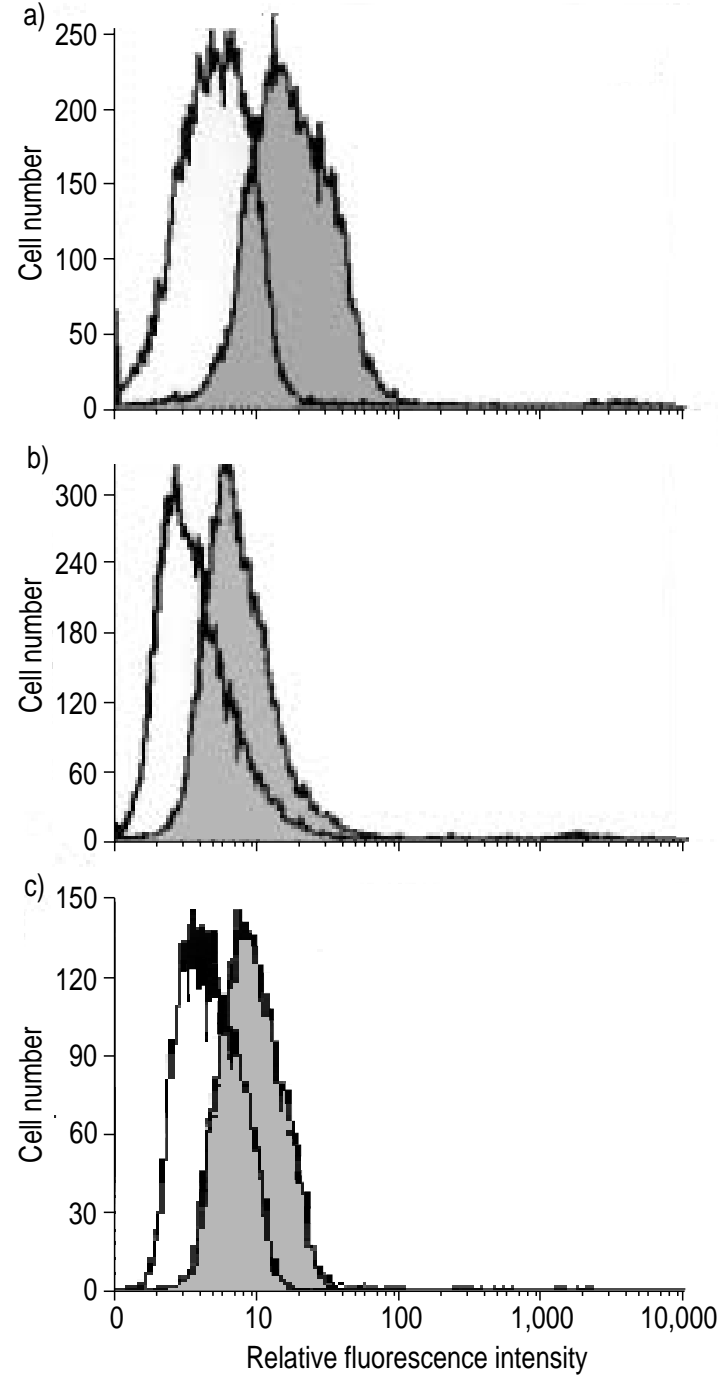

Fig. 2. - Fluorescence-activated cell sorter analyses of discoidin domain receptor 1 (DDR 1 ) expression on human bronchial epithelial (HBE) cells and HBE cell lines with anti-DDR1. Stippled histograms show analyses of a) HBE cells, b) BET-1A cells and c) BEAS-2B cells stained with anti-DDR1. The open histogram shows antigen-peptide blocking.

It was found that the incidence of DDR 1 was highest among the RTKs identified. DDR1 was identified during a search for tyrosine kinase proteins expressed in breast carcinoma cells [8]. This RTK is distinct from other members of the large RTK group because it contains a domain homologous to discoidin, a lectin expressed during the aggregation process of the slime mold $D$. discoideum [15]. cDNAs encoding proteins homologous to DDR1 have been independently cloned from human, mouse, and rat sources by several laboratories [8-14]. DDR1 messenger (RNA mRNA) is expressed in epithelial cells in the brain, gastrointestinal tract, lung, and kidney [8-14].

By Northern analysis, it was shown that DDR1 mRNA is also expressed in HBE cells and two HBE cell lines, BET-1A and BEAS-2B. These lines have been shown to have similar biological properties as HBE 

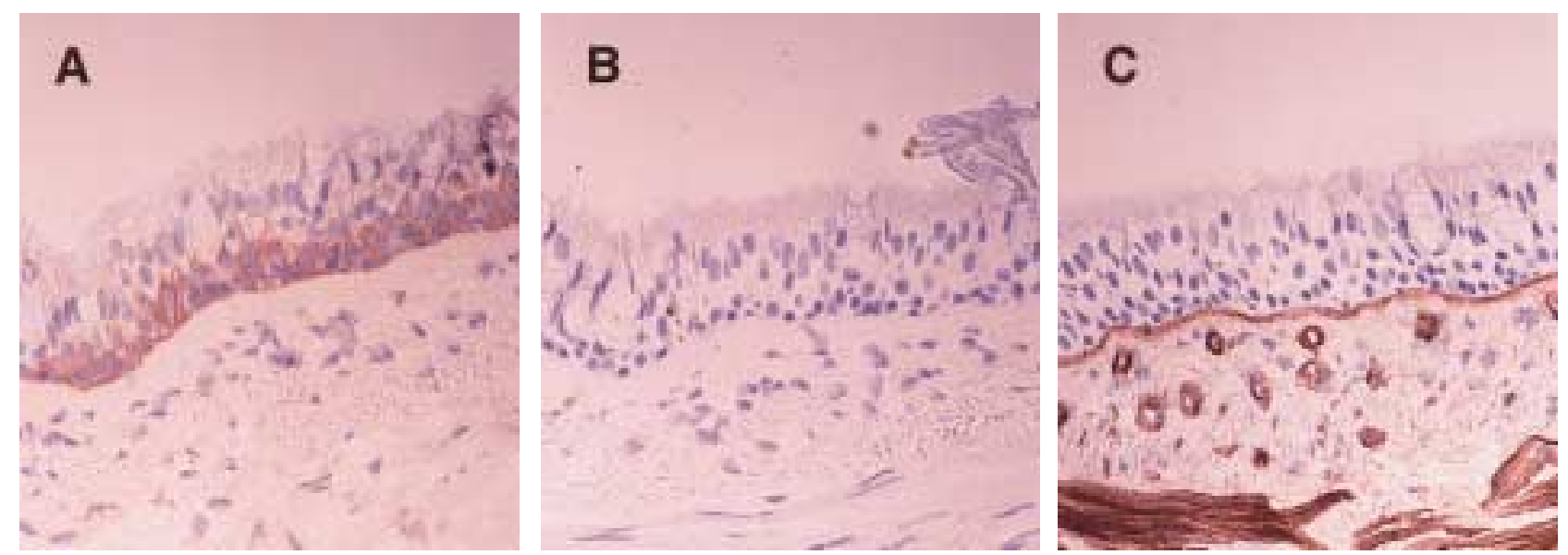

Fig. 3. - Immunolocalization of discoidin domain receptor 1 (DDR1) in the human bronchial epithelium. (A) DDR was $_{1}$ predominantly positive at the basolateral surface of the bronchial epithelium. (B) Positive staining was completely blocked in the presence of an excess of antigen peptide. (C) Immunostaining of type IV collagen in bronchial tissue. Type IV collagen of the basement membrane was closely attached to the basal surface of the bronchial epithelium.

cells $[34,35]$. In addition, FACS analyses showed that DDR 1 protein is expressed in HBE cells and HBE cell lines. Furthermore, immunohistochemical studies demonstrated that DDR1 is predominantly expressed on the basolateral surface of the bronchial epithelium. These findings suggest that DDR1 is involved in a cellular function specific to those cells.

Recently, various types of collagen have been identified as ligands for the "orphan" receptor DDR1 $[19,20]$. Binding of collagen to DDR 1 results in delayed, but sustained, tyrosine kinase activation and autophosphorylation. Based on these observations, it is hypothesized that there is a functional interaction between the basement membrane and DDR 1 expressed on bronchial epithelial cells. The basement membrane is a meshwork of type IV collagen, glycoproteins such as laminin and entactin, and heparin sulphate proteoglycans [36]. Type IV collagen is the structural backbone of the basement membrane. Immunostaining of type IV collagen in bronchial tissues demonstrated that basement membranes are closely attached to the basal surface of the bronchial epithelium, supporting a possible interaction between type IV collagen and DDR 1 expressed on the bronchial epithelium. Alves et al. [14] showed that DDR1 RNA is expressed in the outer epithelial layer of the lung, kidney, and colon in close proximity to the basement membrane, an observation similar to the present findings. The localization of DDR1 to specific subregions of the cell surface may be regulated to mediate collagen signals. To date, however, the biological significance of the interaction of collagen with DDR1 is unknown.

Interestingly, DDR 1 is overexpressed in mammary [37], ovarian [13], and oesophageal carcinomas [38]. More importantly, mutually exclusive expression of DDR1 and the closely related receptor DDR2 has been demonstrated in ovarian and lung carcinomas [14]. DDR1 expression is restricted to epithelial tumour cells, whereas DDR2 mRNA is detected only in the surrounding stromal cells, suggesting that these receptors are involved in tumourigenesis of epithelial carcinomas. Moreover, VoGel et al. [19] have shown that collagen-induced expression of matrix metalloproteinase-1 (MMP-1), an enzyme that specifically cleaves native fibrillar collagen, is mediated by DDR2. These results suggest that overexpression of DDR1 in breast, ovarian, and other epithelial tumours, along with DDR2 expression in the surrounding connective tissues, may be responsible for overexpression of MMP1 in the tumour, which in turn degrades collagen surrounding the tumour and promotes metastasis. The function of DDR1 activation in bronchial epithelial cells is currently under investigation.

In conclusion, it has been shown that a receptor tyrosine kinase, discoidin domain receptor 1 , is predominantly expressed on the basolateral surface of human bronchial epithelium and is colocalized with its ligand type IV collagen. Discoidin domain receptor 1 signalling in bronchial epithelial cells might be essential for differentiation of the cells.

\section{References}

1. Ullrich A, Schlessinger J. Signal transduction by receptors with tyrosine kinase activity. Cell 1990; 61: $203-212$.

2. Schlessinger J, Ullrich A. Growth factor signaling by receptor tyrosine kinases. Neuron 1992; 9: 383-391.

3. Fantl WJ, Johonson DE, Williams LT. Signaling by receptor tyrosine kinases. Annu Rev Biochem 1993; 62: $453-481$.

4. Yarden Y, Ullrich A. Growth factor receptor tyrosine kinases. Annu Rev Biochem 1988; 57: 443-478.

5. Tsao MS, Zhu H, Viallet J. Autocrine growth loop of the epidermal growth factor receptor in normal and immortalized human bronchial epithelial cells. Exp Cell Res 1996; 223: $268-273$.

6. Singh-Kaw P, Zarnegar R, Siegfried JM. Stimulatory effects of hepatocyte growth factor on normal and neoplastic human bronchial epithelial cells. Am J Physiol 1995; 268: L1012 - L1020.

7. Sakamoto O, Iwama A, Amitani R, et al. Role of macrophage-stimulating protein and its receptor, 
RON tyrosine kinase, in ciliary motility. J Clin Invest 1997; 99: $701-709$.

8. Johnson JD, Edman JC, Rutter WJ. A receptor tyrosine kinase found in breast carcinoma cells has an extracellular discoidin I-like domain. Proc Natl Acad Sci USA 1993; 90: 5677-5681.

9. Zerlin M, Julius MA, Goldfarb M. NEP: a novel receptor-like tyrosine kinase expressed in proliferating neuroepithelia. Oncogene 1993; 8: 2731 - 2739.

10. Di Marco E, Cutuli N, Guerra L, Cancedda R, De Luca M. Molecular cloning of trkE, a novel trkrelated putative tyrosine kinase receptor isolated from normal human keratinocytes and widely expressed by normal human tissues. J Biol Chem 1993; 268: 24290 24295.

11. Perez JL, Shen X, Finkernagel S, et al. Identification and chromosomal mapping of a receptor tyrosine kinase with a putative phospholipid binding sequence in its ectodomain. Oncogene 1994; 9: 211-219.

12. Sanchez MP, Tapley P, Saini SS, He B, Pulido D, Barbacid M. Multiple tyrosine protein kinases in rat hippocampal neurons: isolation of Ptk-3, a receptor expressed in proliferative zones of the developing brain. Proc Natl Acad Sci USA 1994; 91: 1819-1823.

13. Laval S, Butler R, Shelling AN, Hanby AM, Poulsom $\mathrm{R}$, Ganesan TS. Isolation and characterization of an epithelial-specific receptor tyrosine kinase from an ovarian cancer cell line. Cell Growth Differ 1994; 5: $1173-1183$

14. Alves F, Vogel W, Mossie K, Millauer B, Hofler H, Ullrich A. Distinct structural characteristics of discoidin I subfamily receptor tyrosine kinases and complementary expression in human cancer. Oncogene 1995; 10: 609-618.

15. Springer WR, Cooper DN, Barondes SH. Discoidin I is implicated in cell-substratum attachment and ordered cell migration of Dictyostelium discoideum and resembles fibronectin. Cell 1984; 39: 557-564.

16. Kane WH, Davie EW. Blood coagulation factors V and VIII: structural and functional similarities and their relationship to hemorrhagic and thrombotic disorders. Blood 1988; 71: 539-555.

17. Soker S, Takashima S, Miao HQ, Neufeld G, Klagsbrun M. Neuropilin-1 is expressed by endothelial and tumor cells as an isoform-specific receptor for vascular endothelial growth factor. Cell 1998; 92: $735-745$.

18. Peles E, Nativ M, Lustig M, et al. Identification of a novel contactin-associated transmembrane receptor with multiple domains implicated in protein-protein interactions. EMBO J 1997; 16: 978 -988.

19. Vogel W, Gish GD, Alves F, Pawson T. The discoidin domain receptor tyrosine kinases are activated by collagen. Mol Cell 1997; 1: 13-23.

20. Shrivastava A, Radziejewski C, Campbell E, et al. An orphan receptor tyrosine kinase family whose members serve as nonintegrin collagen receptors. $\mathrm{Mol}$ Cell 1997; 1: 25-34.

21. Schlessinger $\mathbf{J}$. Direct binding and activation of receptor tyrosine kinases by collagen. Cell 1997; 91: $869-872$.

22. Vogel W. Discoidin domain receptors: structural relations and functional implications. FASEB $J$ 1999. 13 (Suppl): S77-S82.

23. Reddel RR, Ke Y, Gerwin BI, et al. Transformation of human bronchial epithelial cells by infection with SV40 or adenovirus-12 SV40 hybrid virus, or transfection via strontium phosphate coprecipitation with a plasmid containing SV40 early region genes. Cancer Res 1988; 48: 1904-1909.

24. Masui T, Wakefield LM, Lechner JF, LaVeck MA, Sporn MB, Harris CC. Type $\beta$ transforming growth factor is the primary differentiation-inducing serum factor for normal human bronchial epithelial cells. Proc Natl Acad Sci USA 1986; 83: 2438 - 2442.

25. Coleman DL, Tuet IK, Widdicombe JH. Electrical properties of dog tracheal epithelial cells grown in monolayer culture. Am J Physiol 1984; 246: C355C359.

26. Wilks AF. Two putative protein-tyrosine kinases identified by application of the polymerase chain reaction. Proc Natl Acad Sci USA 1989; 86: 1603 1607.

27. Sambrook J, Fritsch EF, Maniatis T. Molecular Cloning. A Laboratory Manual (ed 2). New York, Cold Spring Harbor Laboratory, 1989.

28. Isobe Y, Chen ST, Nakane PK, Brown WR. Studies on translocation of immunoglobulins across intestinal epithelium. I. Improvements in the peroxidase-labeled antibody method for application to study of human intestinal mucosa. Acta Histochem Cytochem 1977; 10: $161-171$.

29. Retsch-Bogart GZ, Stiles AD, Moats-Staats BM, Van Scott MR, Boucher RC, D'Ercole AJ. Canine tracheal epithelial cells express the type 1 insulin-like growth factor receptor and proliferate in response to insulinlike growth factor I. Am J Respir Cell Mol Biol 1990; 3: $227-234$.

30. Kanai M, Rosenberg I, Podolsky DK. Cytokine regulation of fibroblast growth factor receptor $3 \mathrm{IIIb}$ in intestinal epithelial cells. Am J Physiol 1997; 272: G885-G983.

31. Lindberg RA, Hunter T. cDNA cloning and characterization of eck, an epithelial cell receptor proteintyrosine kinase in the eph/elk family of protein kinases. Mol Cell Biol 1990; 10: 6316-6324.

32. Serfas MS, Tyner AL. Ryk is expressed in a differentiation-specific manner in epithelial tissues and is strongly induced in decidualizing uterine stroma. Oncogene 1998; 17: 3435-3444.

33. Nikolova Z, Djonov V, Zuercher G, Andres AC, Ziemiecki A. Cell-type specific and estrogen dependent expression of the receptor tyrosine kinase EphB4 and its ligand ephrin-B2 during mammary gland morphogenesis. J Cell Sci 1998; 111: $2741-2751$.

34. Levine SJ, Larivee P, Logun C, Angus CW, Shelhamer JH. Corticosteroids differentially regulate secretion of IL-6, IL-8, and G-CSF by a human bronchial epithelial cell line. Am J Physiol 1993; 265: L360L368.

35. Bloemen PG, van den Tweel MC, Henricks PA, et al. Expression and modulation of adhesion molecules on human bronchial epithelial cells. Am J Respir Cell Mol Biol 1993; 9: 586-593.

36. Yurchenco PD, Schittny JC. Molecular architecture of basement membranes. FASEB J 1990; 4: 1577-1590.

37. Barker KT, Martindale JE, Mitchell PJ, et al. Expression patterns of the novel receptor-like tyrosine kinase, DDR, in human breast tumours. Oncogene 1995; 10: $569-575$.

38. Nemoto $\mathrm{T}$, Ohashi K, Akashi T, Johnson JD, Hirokawa K. Overexpression of protein tyrosine kinases in human esophageal cancer. Pathobiology 1997; 65: 195-203. 